\title{
Paraneoplastic Polymyalgia Rheumatica as Manifestation of a Pancreatic Neuroendocrine Tumour
}

\author{
Luís Filipe Linhares Santos ${ }^{a}$, Cláudia Susana Teixeira Graça Fernandes ${ }^{\text {, }}$, António Pedro Mendes Duarte Rodrigues Teotónio ${ }^{a}$
}

a Department of Internal Medicine, Coimbra University Hospital, Coimbra, Portugal

${ }^{b}$ Department of Infectious Diseases, Coimbra University Hospital, Coimbra, Portugal

\begin{abstract}
Autoimmune diseases may present as paraneoplastic syndrome. This is especially recognized in the case of polymyositis/dermatomyositis, but is less common in polymyalgia rheumatica. The authors describe the case of a 73-year-old man who presented with pain and stiffness of the scapular and pelvic girdles associated with asthenia lasting for a few weeks. The presence of therapeutic resistance and other atypical features directed the investigation towards the search of an occult malignancy. Patient evaluation revealed a pancreatic neuroendocrine tumour. After surgical treatment of the underlying neoplasia, the patient recovered fully with resolution of the rheumatic disease.
\end{abstract}

Keywords: Paraneoplastic syndrome, polymyalgia rheumatica, cancer

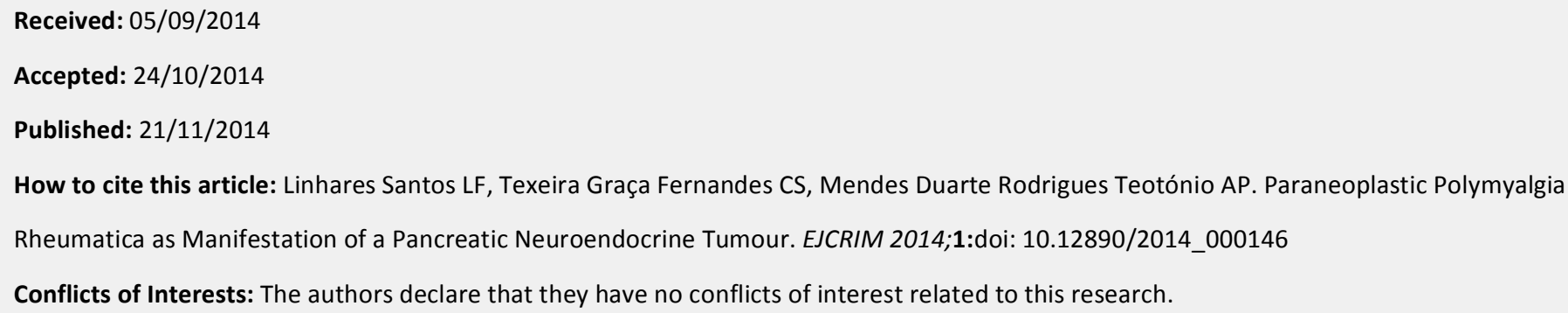

\section{Introduction}

Paraneoplastic syndrome (PNS) is defined by signs or symptoms related to a tumour without a direct anatomical relationship with it, not explained by metastatic disease [1].

Polymyalgia rheumatica (PMR) is an inflammatory condition belonging to the connective tissue diseases, which occurs quite frequently in the elderly. In case of atypical manifestations or the absence of an adequate response to treatment, it should trigger an extensive aetiological investigation. There have been a few cases that report an association with malignant tumours in a synchronous fashion or prior to the appearance of the cancer. 


\section{European Journal}

of Case Reports in

Internal Medicine

Pancreatic neuroendocrine tumours represent a small percentage of pancreatic neoplasms (1\%-2\%) [2]. Diagnosis of neuroendocrine tumours is very difficult, especially if the tumours are small and non-functional.

The authors report a rare case of PMR as a paraneoplastic manifestation of a pancreatic neuroendocrine neoplasm.

\section{Case report}

A 73-year-old man was admitted to the emergency department (ED) complaining of symmetrical aching and stiffness of the scapular and pelvic girdles, which had begun a few weeks earlier. The patient also described asthenia, pain of the wrists and morning stiffness. There were no other associated complaints.

He had already been medicated with diclofenac and etoricoxib without improvement.

The patient had no clinical records and was not taking any chronic medication.

The physical examination showed bilateral tenderness of the muscles of the arms, shoulders and hips in the absence of peripheral arthritis. He was unable to stand up from a chair without help. There were no other remarkable findings on clinical examination.

Laboratory tests revealed a mild normocytic anaemia $(\mathrm{Hb} 12.1 \mathrm{~g} / \mathrm{dl}$ ), elevated erythrocyte sedimentation rate (ESR) of $93 \mathrm{~mm} / \mathrm{h}$ and C-reactive protein (CRP) of $17.4 \mathrm{mg} / \mathrm{dl}$. Rheumatoid factor and anti-citrullinated protein antibody were both negative as well as the antinuclear antibodies. Tumour markers were also negative. Hand X-rays showed lesions of the proximal and distal interphalangeal joints, suggestive of degenerative changes (Fig. 1). Chest X-ray was normal.

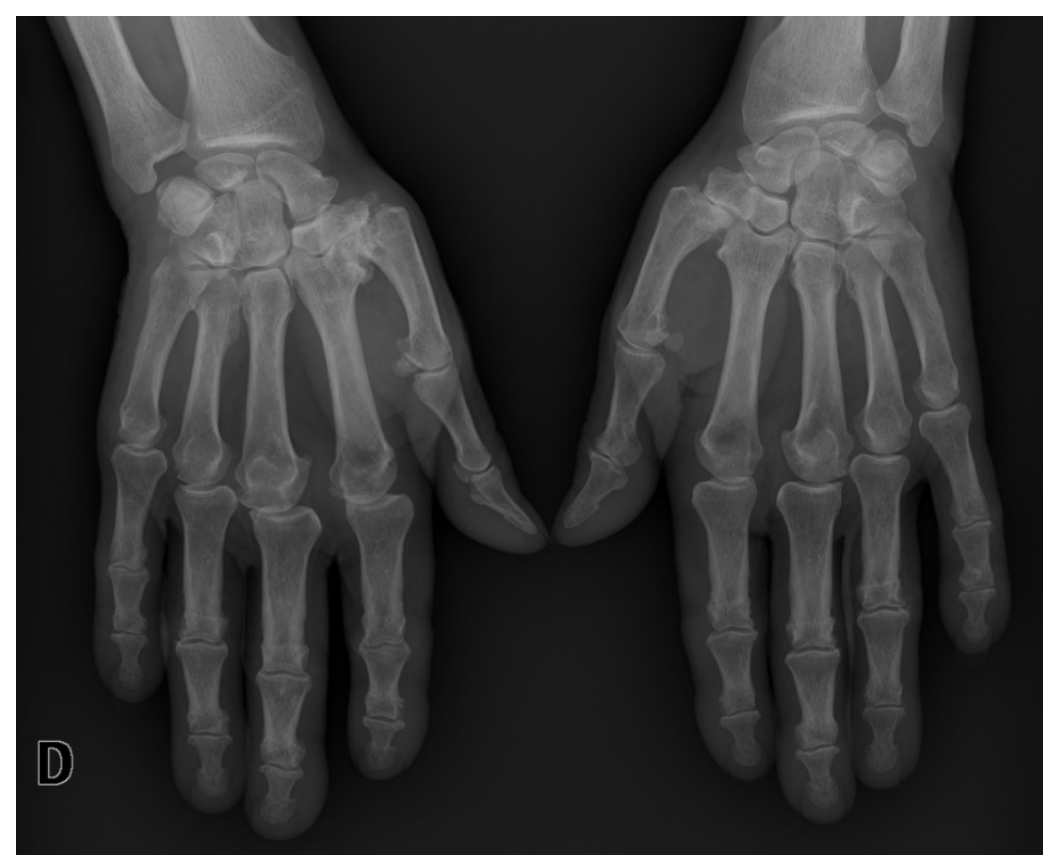

Figure 1: Hand X-ray showing degenerative changes in both hands.

Using the provisional classification criteria for PMR previously published [3], a hypothesis of PMR was assumed. The patient was started on prednisolone $20 \mathrm{mg}$ daily. 


\section{European Journal}

of Case Reports in

Internal Medicine

Three weeks later, only slight clinical improvement was achieved and ESR remained elevated $(74 \mathrm{~mm} / \mathrm{h})$. The patient also presented new complaints of headache and induration of the temporal artery. A temporal artery biopsy was performed and prednisolone was increased to $60 \mathrm{mg}$ daily $(1 \mathrm{mg} / \mathrm{kg} /$ day $)$ pending biopsy results.

In 4 weeks, the inflammatory markers decreased (ESR to $10 \mathrm{~mm} / \mathrm{h}, \mathrm{CRP}<0.5 \mathrm{mg} / \mathrm{dl}$ ), anaemia improved ( $\mathrm{Hb} 13.4 \mathrm{~g} / \mathrm{dl}$ ), and no other laboratory changes were identified. However, clinically he still had not fully recovered, reporting pain and stiffness of the scapular and pelvic girdles.

Biopsy showed no histological findings of giant cell arteritis. Prednisolone was readjusted to the initial dose, and a subsequent investigation was undertaken.

The CT scan of the thorax, abdomen and pelvis revealed a thrombosed segment of the splenic vein (Fig. 2) and abdominal MRI showed a tumour of the pancreatic tail, prolapsing to and compressing the adjacent segment of the splenic vein (Fig. 3).

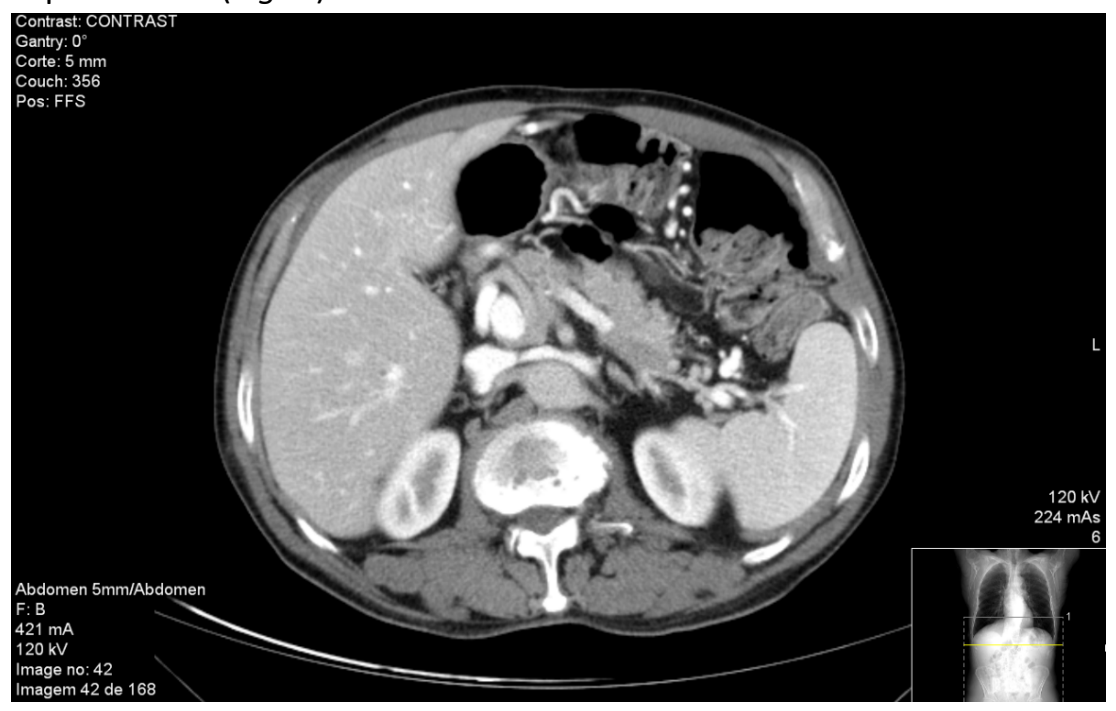

Figure 2: Contrast-enhanced CT scan showing thrombosed segment of the splenic vein approximately $4 \mathrm{~cm}$ in length.

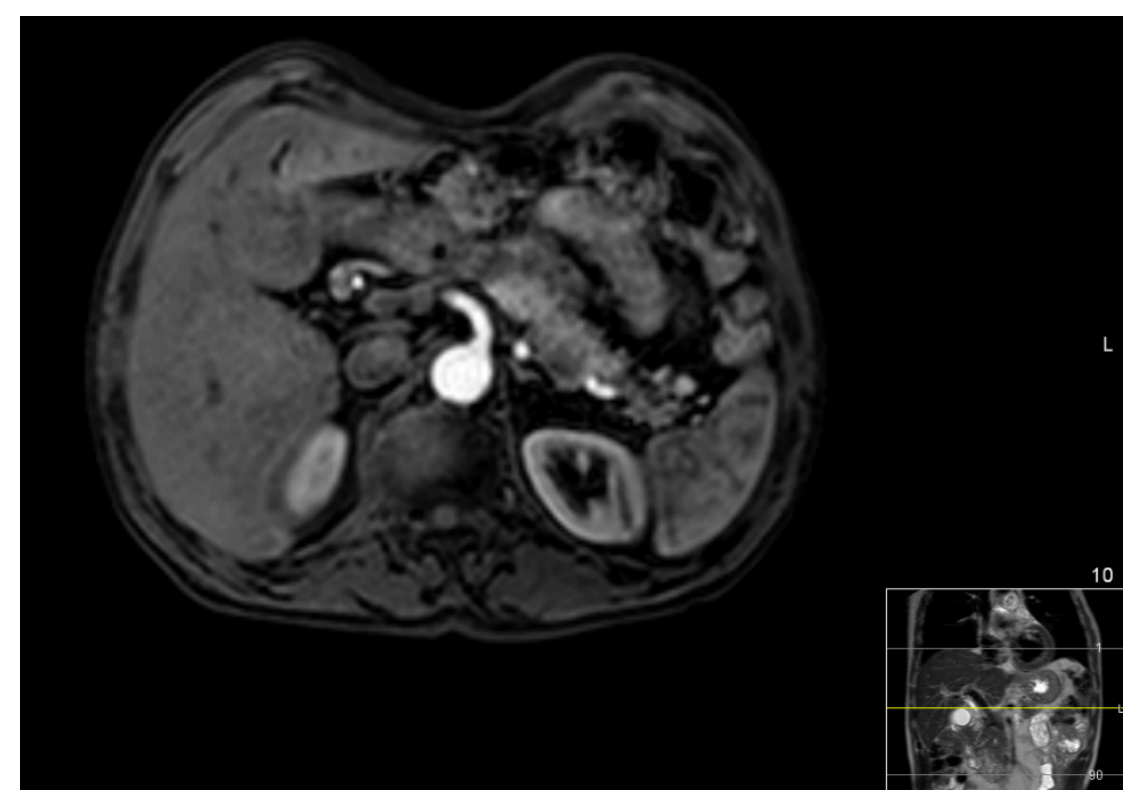

Figure 3: Magnetic resonance imaging (SPAIR sequence) showing tumour lesion of the pancreas. 
The patient was referred to the surgery department and was submitted to a distal pancreatectomy with splenectomy. Histopathology revealed a well-differentiated pancreatic neuroendocrine neoplasm, Ki-67 of 10\%, staged as pT3 NO, with no metastatic disease identified on the 11 excised peripancreatic lymph nodes.

Apart from secondary diabetes mellitus, the overall outcome was favourable. There was no recurrence of clinical symptoms of pain or stiffness and so it was decided to discontinue prednisolone. The patient has been symptom free for 18 months after surgery with no evidence of relapse of the neoplastic disease.

\section{Discussion}

Autoimmune diseases can be triggered by tumour development, commonly referred to as PNS.

These can emerge after onset of tumours or metastases, simultaneous to the neoplasm diagnosis, or they can be the cancer's first manifestation [1].

Polymyositis and dermatomyositis are the most commonly described rheumatic disorders associated with PNS [1]. PMR association with PNS is still controversial, but a few reports have established connections with lung [4] and colon [5] adenocarcinoma.

Neuroendocrine tumours (NETs) are considered a rare type of neoplasm. They are classified as functional or non-functional according to the presence or absence, respectively, of clinical symptoms associated with the production and secretion of various neuroendocrine mediators. Only $10 \%$ of NETs are functional [2].

When the symptoms are unusual or the tumour is non-functional, the diagnosis becomes more challenging. Recognizing the existence of such PNS is important as, if not identified, it may delay the neoplasia diagnosis [6].

The absence of a sustained clinical response to treatment led us to expand our investigation. The hypothesis of a concomitant giant cell arteritis was assumed, but lack of complete clinical response to corticosteroid increment together with negative results of the temporal artery biopsy excluded this diagnosis, directing the investigational study towards an eventual associated neoplasia.

The study revealed a pancreatic neuroendocrine tumour.

The relationship between the rheumatic syndrome and cancer was reinforced by the fact that the treatment of the underlying tumour led to a clear improvement of the rheumatic symptoms, enabling the discontinuation of corticotherapy.

\section{Learning Points}

- It is critical to recognize the presence of a PNS as it may lead to the diagnosis of an unsuspected underlying neoplasm and be useful in the follow-up of the underlying disease.

- Whenever a case of PMR exhibits therapeutic resistance or other atypical features, prompt investigation should be carried out since it may mask a more threatening disease. 


\section{European Journal}

of Case Reports in

Internal Medicine

\section{References}

1. Fam AG. Paraneoplastic rheumatic syndrome, Baillieres Best Pract Res Clin Rheumatol 2000;14:515-533.

2. Milan SA, Yeo CJ. Neuroendocrine tumors of the pancreas, Curr Opin Oncol 2012;24:46-55.

3. Dasgupta B, Cimmino M, Kremers H, Schmidt W, Schirmer M, Salvarani C et al. 2012 Provisional classification criteria for polymyalgia rheumatica: a European League Against Rheumatism/American College of Rheumatology collaborative initiative, Arthritis Rheum 2012;64:943-954.

4. Cocquempot K, Defuentes G, Duron-Martineau S, Berets O, Vaylet F, Margery J. Polymyalgia rheumatica revealing a lung cancer, Rev Mal Respir 2013;30:67-70.

5. Kehler T, Curković B. Polymyalgia rheumatica and colon malignancy: case report. Clin Rheumatol 2006;25:764765.

6. Kaltsas G, Androulakis II, Herder WW, Grossman AB. Paraneoplastic syndromes secondary to neuroendocrine tumours, Endocr Relat Cancer 2010;17:R173-R193. 